\title{
Alamouti-Type Space-Time Coding for Free-Space Optical Communication with Direct Detection
}

\author{
M. K. Simon ${ }^{1}$ and V. Vilnrotter ${ }^{1}$
}

\begin{abstract}
In optical communication systems employing direct detection at the receiver, intensity modulations such as on-off keying (OOK) or pulse-position modulation (PPM) are commonly used to convey the information. Consider the possibility of applying space-time coding in such a scenario, using, for example, an Alamouti-type coding scheme [1]. Implicit in the Alamouti code is the fact that the modulation that defines the signal set is such that it is meaningful to transmit and detect both the signal and its negative. While modulations such as phase-shift keying (PSK) and quadrature amplitude modulation (QAM) naturally fall into this class, $O O K$ and PPM do not since the signal polarity (phase) would not be detected at the receiver. We investigate a modification of the Alamouti code to be used with such modulations that has the same desirable properties as the conventional Alamouti code but does not rely on the necessity of transmitting the negative of a signal.
\end{abstract}

\section{System Model}

Consider an optical communication system with two transmit antennas and one receive antenna as shown in Fig. 1. This model inherently assumes that both transmitters are simultaneously observed by the receiver, thus implying a large receiver field of view (FOV). This, in turn, implies a larger collected background radiation that could seriously degrade system performance. Therefore, care must be exercised to limit background through the use of appropriate spatial filtering prior to detection. For example, if the link distance is $R \mathrm{~m}$ and the receiver FOV is $\theta \mathrm{rad}$, then the separation of the two transmitters cannot exceed approximately $\theta R \mathrm{~m}$. Thus, if $R=1 \mathrm{~km}$ and $\theta \cong 10 \mathrm{mrad}$, then the transmitters would have to be less than $10 \mathrm{~m}$ apart for the scheme to work. This model is applicable to urban "last mile" links where multiple transmitters and receivers can be envisioned for mitigating link outage due to turbulence, scattering, and occasional blockage.

\section{The Modified Alamouti Code}

The channel gain from antenna $j$ to the single receive antenna is denoted by $h_{1 j}, j=1,2$, and is assumed to be constant over a two-symbol duration. In the conventional Alamouti code, in the first bit interval, antenna 1 transmits signal $x_{1}$ and antenna 2 transmits $x_{2}$, where $x_{1}$ and $x_{2}$ each range over

\footnotetext{
${ }^{1}$ Communications Systems and Research Section.

The research described in this publication was carried out by the Jet Propulsion Laboratory, California Institute of Technology, under a contract with the National Aeronautics and Space Administration.
} 


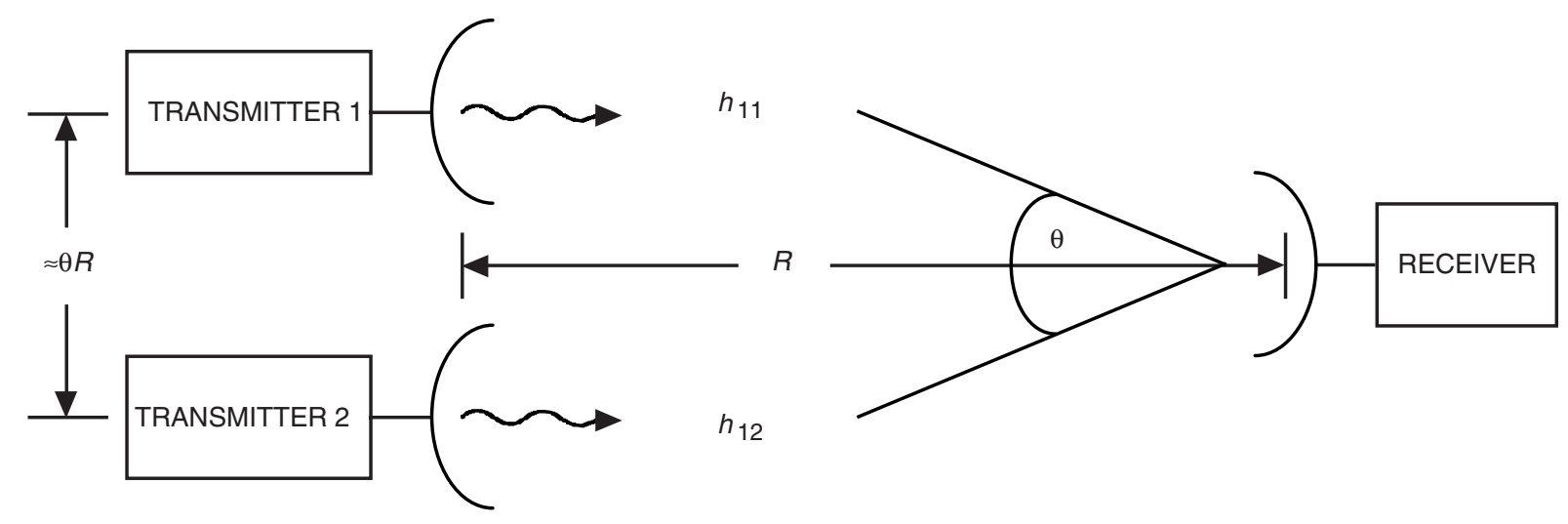

Fig. 1. Optical communication link.

the signal set $\left(s_{1}, s_{2}\right)$. In the second bit interval, antenna 1 transmits $-x_{2}$ whereas antenna 2 transmits $x_{1}$. Note that here we are dealing with real signals and thus the complex conjugate notation that normally appears on the $x_{i}$ 's transmitted in the second symbol interval is absent. A likewise statement applies to the channel gains which, in the application under consideration, represent the modification of the amplitude of the path from the optical source, e.g., a laser, to the photodetector - the phase modulation associated with the fading process disappears as a result of the direct detection. Furthermore, the channel gains, which here represent fading intensity, have statistical models equivalent to the magnitude squared of the channel gains in the RF problem.

We consider the channel model commonly employed in ground-based free-space optical links where high signal energies allow the approximation of the Poisson photon-counting detection model by a much simpler continuous Gaussian limiting form. More specifically, we consider the case of binary communication in an additive white Gaussian noise (AWGN) background which occurs, for example, in a laser communication scenario where the signal energy is so great that the receiver signal-to-noise ratio (SNR) is limited by shot noise inherent to the photodetection process itself. System behavior in such a scenario is referred to as a photon-limited or shot-noise-limited operation [2, Chapter 7, and 3].

In the case of on-off keying (OOK), the signals are described by the waveforms

$$
\left.\begin{array}{l}
s_{1}=0, \quad 0 \leq t \leq T \\
s_{2}=A, \quad 0 \leq t \leq T
\end{array}\right\}
$$

where $A$ is a positive constant related to the intensity of the light source and $T$ is the signal duration. In the case of binary pulse-position modulation (PPM), the signals are described by the waveforms

$$
\begin{gathered}
s_{1}= \begin{cases}0, & 0 \leq t \leq \frac{T}{2} \\
A, & \frac{T}{2}<t \leq T \\
s_{2}=\left\{\begin{array}{ll}
A, & 0 \leq t \leq \frac{T}{2} \\
0, & \frac{T}{2}<t \leq T
\end{array}\right\}\end{cases}
\end{gathered}
$$


Note that, for either modulation, we can express one of these signal waveforms in terms of the other by

$$
s_{i}=-s_{j}+A, \quad j \neq i
$$

Using an overbar notation, we define the complement of a signal $x_{i}$ by $\bar{x}_{i}$, where what is meant by complement is the signal waveform obtained by reversing the roles of "on" and "off." For example,

$$
\begin{aligned}
& \text { if } x_{i}=s_{1}, \\
& \text { then } \bar{x}_{i} \triangleq s_{2}=-s_{1}+A=-x_{i}+A
\end{aligned}
$$

Likewise,

$$
\begin{aligned}
& \text { if } x_{i}=s_{2}, \\
& \text { then } \bar{x}_{i} \triangleq s_{1}=-s_{2}+A=-x_{i}+A
\end{aligned}
$$

As in the conventional Alamouti code, in the first bit interval, antenna 1 transmits signal $x_{1}$ and antenna 2 transmits $x_{2}$, where again $x_{1}$ and $x_{2}$ each range over the signal set $\left(s_{1}, s_{2}\right)$, now chosen specifically in accordance with Eq. (1) or Eq. (2). In the second bit interval, however, antenna 1 transmits $\bar{x}_{2}$ whereas antenna 2 transmits $x_{1}$. Again we emphasize the point that in the case of OOK or PPM we are dealing with real signals; hence, it is not necessary to associate any complex conjugate notation with $x_{1}$ and $x_{2}$ as is done in the conventional Alamouti code.

Based on the above communication model, the received signals in the first and second bit intervals, respectively, are given by

$$
\left.\begin{array}{l}
y_{1}=h_{11} x_{1}+h_{12} x_{2}+n_{1} \\
y_{2}=h_{11} \bar{x}_{2}+h_{12} x_{1}+n_{2}
\end{array}\right\}
$$

or, equivalently,

$$
\left.\begin{array}{c}
y_{1}=h_{11} x_{1}+h_{12} x_{2}+n_{1} \\
y_{2}-h_{11} A=-h_{11} x_{2}+h_{12} x_{1}+n_{2}
\end{array}\right\}
$$

where $n_{1}, n_{2}$ are modeled as independent zero-mean Gaussian real random variables (RVs) with variance $\sigma^{2}$.

Assuming perfect knowledge of the channel gains and the transmitted signal level, ${ }^{2}$ the receiver uses the sequence $y_{1}, y_{2}$ to construct

${ }^{2}$ Knowledge of the transmitted signal level at the receiver may not be viewed as an additional requirement since for OOK such knowledge is necessary to set the detection threshold. 


$$
\left.\begin{array}{l}
\tilde{x}_{1}=h_{11} y_{1}+h_{12} y_{2}-h_{11} h_{12} A \\
\tilde{x}_{2}=h_{12} y_{1}-h_{11} y_{2}+h_{11}^{2} A
\end{array}\right\}
$$

Substituting Eq. (6) into Eq. (8) results in

$$
\begin{aligned}
\tilde{x}_{1} & =h_{11}\left(h_{11} x_{1}+h_{12} x_{2}+n_{1}\right)+h_{12}\left(h_{11} \bar{x}_{2}+h_{12} x_{1}+n_{2}\right)-h_{11} h_{12} A \\
& =\left(h_{11}^{2}+h_{12}^{2}\right) x_{1}+h_{11} h_{12}\left(x_{2}+\bar{x}_{2}-A\right)+h_{11} n_{1}+h_{12} n_{2} \\
\tilde{x}_{2} & =h_{12}\left(h_{11} x_{1}+h_{12} x_{2}+n_{1}\right)-h_{11}\left(h_{11} \bar{x}_{2}+h_{12} x_{1}+n_{2}\right)+h_{11}^{2} A \\
& =h_{11}^{2}\left(A-\bar{x}_{2}\right)+h_{12}^{2} x_{2}+h_{12} n_{1}-h_{11} n_{2}
\end{aligned}
$$

However, from Eqs. (4) and (5), $\bar{x}_{2}=-x_{2}+A$. Using this in Eq. (9) gives

$$
\left.\begin{array}{l}
\tilde{x}_{1}=\left(h_{11}^{2}+h_{12}^{2}\right) x_{1}+h_{11} n_{1}+h_{12} n_{2}=\left(h_{11}^{2}+h_{12}^{2}\right) x_{1}+\tilde{N}_{1} \\
\tilde{x}_{2}=\left(h_{11}^{2}+h_{12}^{2}\right) x_{2}+h_{12} n_{1}-h_{11} n_{2}=\left(h_{11}^{2}+h_{12}^{2}\right) x_{2}+\tilde{N}_{2}
\end{array}\right\}
$$

which is the set of decision metrics identical to those in the conventional Alamouti code. Thus, the modified Alamouti code as described above produces a diversity of order 2 and partitioning of the maximumlikelihood (ML) decision so as to provide the necessary independence to allow each symbol to be detected separately. ${ }^{3}$

The above modification also can be applied to the more general (not necessarily binary) PPM case. Here the $i$ th signal in the set is modeled as the waveform

$$
s_{i}= \begin{cases}A, & \frac{(i-1) T}{M} \leq t \leq \frac{i T}{M}, \quad i=1,2, \cdots, M \\ 0, & \text { otherwise }\end{cases}
$$

and, analogous to the binary case, its complement would be defined as

$$
\bar{s}_{i}= \begin{cases}0, & \frac{(i-1) T}{M} \leq t \leq \frac{i T}{M}, \quad i=1,2, \cdots, M \\ A, & \text { otherwise }\end{cases}
$$

Since from Eqs. (11) and (12) we have $\bar{s}_{i}=-s_{i}+A, i=1,2, \cdots, M$, then analogously to Eqs. (4) and (5) we would have $\bar{x}_{2}=-x_{2}+A$, which when used in Eq. (9) once again produces Eq. (10).

\footnotetext{
${ }^{3}$ Note that the noise samples $\tilde{N}_{1}$ and $\tilde{N}_{2}$ are uncorrelated and, since they are complex Gaussian, they are also independent.
} 


\section{The Maximum-Likelihood Decision Metric}

Based on the observations $y_{1}, y_{2}$, the ML metric for the Gaussian model is proportional to the conditional probability density whose natural logarithm is given by (ignoring terms that are hypothesisindependent)

$$
\begin{aligned}
m(\mathbf{y}, \mathbf{x})= & {\left[y_{1}-\left(h_{11} x_{1}+h_{12} x_{2}\right)\right]^{2}+\left[y_{2}-\left(h_{11} \bar{x}_{2}+h_{12} x_{1}\right)\right]^{2} } \\
= & y_{1}^{2}+y_{2}^{2}-2\left[\left(h_{11} y_{1}+h_{12} y_{2}\right) x_{1}\right]-2\left[\left(h_{12} y_{1}-h_{11} y_{2}\right) x_{2}\right] \\
& -2\left[h_{11} y_{2}\left(\bar{x}_{2}+x_{2}\right)\right]+\left(h_{11}^{2}+h_{12}^{2}\right) x_{1}^{2}+\left(h_{11}^{2}+h_{12}^{2}\right) x_{2}^{2} \\
& +h_{11}^{2}\left(\bar{x}_{2}^{2}-x_{2}^{2}\right)+2\left[h_{11} h_{12} x_{1}\left(\bar{x}_{2}+x_{2}\right)\right]
\end{aligned}
$$

Using Eq. (8), this can be written as

$$
\begin{aligned}
m(\mathbf{y}, \mathbf{x})= & y_{1}^{2}+y_{2}^{2}-2\left[\left(\tilde{x}_{1}+h_{11} h_{12} A\right) x_{1}\right]-2\left[\left(\tilde{x}_{2}-h_{11}^{2} A\right) x_{2}\right] \\
& -2\left(h_{11} y_{2} A\right)+\left(h_{11}^{2}+h_{12}^{2}\right) x_{1}^{2}+\left(h_{11}^{2}+h_{12}^{2}\right) x_{2}^{2} \\
& +h_{11}^{2}\left(A^{2}-2 A x_{2}\right)+2\left(h_{11} h_{12} x_{1} A\right) \\
= & y_{1}^{2}+y_{2}^{2}-2 \tilde{x}_{1} x_{1}-2 \tilde{x}_{2} x_{2}-2\left(h_{11} y_{2} A\right) \\
& +\left(h_{11}^{2}+h_{12}^{2}\right)\left(x_{1}^{2}+x_{2}^{2}\right)+h_{11}^{2} A^{2} \\
= & y_{1}^{2}+y_{2}^{2}-\tilde{x}_{1}^{2}-\tilde{x}_{2}^{2}+\left(\tilde{x}_{1}-x_{1}\right)^{2}+\left(\tilde{x}_{2}-x_{2}\right)^{2}-2\left(h_{11} y_{2} A\right) \\
& +\left(h_{11}^{2}+h_{12}^{2}-1\right)\left(x_{1}^{2}+x_{2}^{2}\right)+h_{11}^{2} A^{2}
\end{aligned}
$$

Eliminating terms from Eq. (14) that are hypothesis-independent, then for the purpose of decision making the metric simplifies to

$$
m(\tilde{\mathbf{x}}, \mathbf{x})=\left(\tilde{x}_{1}-x_{1}\right)^{2}+\left(h_{11}^{2}+h_{12}^{2}-1\right) x_{1}^{2}+\left(\tilde{x}_{2}-x_{2}\right)^{2}+\left(h_{11}^{2}+h_{12}^{2}-1\right) x_{2}^{2}
$$

which is identical to that for the conventional Alamouti code. Finally, since the pair of information signals $x_{1}$ and $x_{2}$ to be transmitted is independently chosen from the binary signal alphabet, then an ML decision can be made separately on each of them using the metric

$$
m\left(\tilde{x}_{i}, x_{i}\right)=\left(\tilde{x}_{i}-x_{i}\right)^{2}+\left(h_{11}^{2}+h_{12}^{2}-1\right) x_{i}^{2}, \quad i=1,2
$$

with the corresponding decision rule: 
Choose $x_{i}=\hat{x}_{i}$ iff

$$
\left(\tilde{x}_{i}-\hat{x}_{i}\right)^{2}+\left(h_{11}^{2}+h_{12}^{2}-1\right) \hat{x}_{i}^{2} \leq\left(\tilde{x}_{i}-x_{i}\right)^{2}+\left(h_{11}^{2}+h_{12}^{2}-1\right) x_{i}^{2}
$$

for $x_{i} \neq \hat{x}_{i}$

In conclusion, as is well-known for the conventional Alamouti code used in the RF application, for the perfectly known channel, the modified Alamouti scheme is once again optimal in the ML sense. Note that this optimality statement is independent of the statistics of the channel since the proof of optimality is based on the conditional (on the channel gains) likelihood function. The performance of the modified Alamouti scheme, however, will be very much dependent on the statistics [probability density functions (PDFs)] used to model the channel gains. Having said this, we point out to the reader a significant difference between the RF and optical applications. Since, for direct detection of OOK or PPM, the received signal current is proportional to the square of the optical field, the models most typically used for the magnitude of the channel gains, i.e., $\left|h_{1 j}\right|, j=1,2$, which are associated with irradiance and arise from atmospheric-induced turbulence, are those that are normally associated with the squared magnitude of the channel gain in the RF application, e.g., negative exponential or gamma-gamma distributions. ${ }^{4}$

\section{Extension to More than One Receive Antenna}

Suppose now that the system contains $L_{r}$ receive antennas. As such, the generalization of Eq. (6) becomes

$$
\begin{aligned}
& y_{l}=h_{l 1} x_{1}+h_{l 2} x_{2}+n_{l}, \\
& y_{l+L_{r}}=h_{l 1} \bar{x}_{2}+h_{l 2} x_{1}+n_{l+L_{r}}, \\
& \left.l=1,2, \cdots, L_{r} \quad\right\}
\end{aligned}
$$

where $y_{l}, l=1,2, \cdots, L_{r}$, represents the signals received by the $L_{r}$ receive antennas in the first bit interval and $y_{l+L_{r}}, l=1,2, \cdots, L_{r}$, represents the signals received by the $L_{r}$ receive antennas in the second bit interval corresponding to a single transmission of the Alamouti code. At the receiver, we now form [analogous to Eq. (8)] the decision statistics

$$
\left.\begin{array}{l}
\tilde{x}_{1}=\sum_{l=1}^{L_{r}} h_{l 1} y_{l}+\sum_{l=1}^{L_{r}} h_{l 2} y_{l+L_{r}}-A \sum_{l=1}^{L_{r}} h_{l 1} h_{l 2} \\
\tilde{x}_{2}=\sum_{l=1}^{L_{r}} h_{l 1} y_{l}-\sum_{l=1}^{L_{r}} h_{l 1} y_{l+L_{r}}+A \sum_{l=1}^{L_{r}} h_{l 1}^{2}
\end{array}\right\}
$$

Substituting Eq. (18) into Eq. (19) and again making use of the property $\bar{x}_{2}=-x_{2}+A$ results in

\footnotetext{
${ }^{4}$ Note that we have intentionally avoided the use of the term " $K$-distribution" to describe the gamma-gamma distribution since the use of this terminology is inconsistent in the literature in that researchers dealing with RF wireless communications in the presence of fading often refer to the Rayleigh-gamma distribution as a $K$-distribution [4].
} 


$$
\left.\begin{array}{l}
\tilde{x}_{1}=\sum_{l=1}^{L_{r}}\left(h_{l 1}^{2}+h_{l 2}^{2}\right) x_{1}+\sum_{l=1}^{L_{r}}\left(h_{l 1} n_{l}+h_{l 2} n_{l+L_{r}}\right)=\sum_{l=1}^{L_{r}}\left(h_{11}^{2}+h_{12}^{2}\right) x_{1}+\tilde{N}_{1} \\
\tilde{x}_{2}=\sum_{l=1}^{L_{r}}\left(h_{l 1}^{2}+h_{l 2}^{2}\right) x_{2}+\sum_{l=1}^{L_{r}}\left(h_{l 2} n_{l}-h_{l 1} n_{l+L_{r}}\right)=\sum_{l=1}^{L_{r}}\left(h_{11}^{2}+h_{12}^{2}\right) x_{2}+\tilde{N}_{2}
\end{array}\right\}
$$

which are the identical statistics achieved by the conventional Alamouti code and demonstrates the achievement of a diversity of order $2 L_{r}$ as well as the ability to detect each symbol separately.

\section{A More Symmetric Solution}

By inspection of Eq. (19), we observe that the bias terms (in one case, the sum of the product of the channel gains from both transmit antennas, and in the other case, the sum of the squares of the channel gains associated with only the first transmit antenna) included in the modified Alamouti decision variables are different for $\tilde{x}_{1}$ and $\tilde{x}_{2}$. Thus, one anticipates that, in the presence of imperfect channel state knowledge, the error-probability performance associated with decisions on $x_{1}$ and $x_{2}$ might tend to favor one over the other. To produce a more symmetric decision mechanism, one can alternate transmissions between two equivalent Alamouti codes. The first code is as previously described. For the second code, the symbols sent in the first bit interval from each antenna remain the same. However, in the second bit interval, antenna 1 transmits $x_{2}$ whereas antenna 2 transmits $\bar{x}_{1}$, i.e., the roles of $x_{1}$ and $x_{2}$ trade places. In this case, analogous to Eq. (6), the received signals in the first and second bit intervals, respectively, are given by

$$
\left.\begin{array}{l}
y_{1}=h_{11} x_{1}+h_{12} x_{2}+n_{1} \\
y_{2}=h_{11} x_{2}+h_{12} \bar{x}_{1}+n_{2}
\end{array}\right\}
$$

Likewise, the receiver now uses the sequence $y_{1}, y_{2}$ to construct

$$
\left.\begin{array}{l}
\tilde{x}_{1}=h_{11} y_{1}-h_{12} y_{2}+h_{12}^{2} A \\
\tilde{x}_{2}=h_{12} y_{1}+h_{11} y_{2}-h_{11} h_{12} A
\end{array}\right\}
$$

Substituting Eq. (21) into Eq. (22) once again produces the identical decision variables as in Eq. (10). Thus, by alternating between the two codes, the effect of channel estimation errors in the bias terms tends to become balanced, thereby not favoring one symbol over the other. A similar construction can be applied in the case of more than one receive antenna.

\section{Error-Probability Performance Evaluation}

Since the decision statistics of the modified Alamouti code are identical to those of the conventional one, it is straightforward to evaluate the error-probability performance of space-time-coded (STC) optical systems by using the analytical tools developed for the RF wireless applications in, for example, [5]. The primary differences between the two performance evaluations are (1) an additional 3-dB SNR loss relative to binary phase-shift keying (BPSK) (for the same average transmitted energy) attributed to the fact that OOK and binary PPM are orthogonal (as opposed to antipodal) modulations, and (2) the statistical modeling of the channel-gain magnitudes in the optical application is that which is normally associated 
with the squared magnitude of the channel gain in the RF application, as mentioned previously. For example, for a single receive antenna STC system using the modified Alamouti code and either uncoded OOK or binary PPM, the conditional bit-error probability (BEP) is given by

$$
P_{b}(E \mid \mathbf{I})=Q\left(\sqrt{\frac{E_{b}}{2 N_{0}}\left(I_{11}^{2}+I_{12}^{2}\right)}\right)
$$

where $Q(x)$ is the Gaussian $Q$-function with argument $x$ and $E_{b} / N_{0}$ is the total bit-energy-to-noise spectral density ratio ${ }^{5}$ per transmit antenna in the absence of turbulence (in OOK, $E_{b}$ represents the energy of the detected pulse in the "on" state whereas, in binary PPM, $E_{b} / 2$ represents the pulse energy in either time slot). Assuming a negative exponential model for the independent identically distributed (i.i.d.) channel irradiances $I_{1 j}=\left|h_{1 j}\right|, j=1,2$, as is often done as a limiting case of strong turbulence conditions [2], given by

$$
p_{I}(I)=\frac{1}{\bar{I}} \exp \left(-\frac{I}{\bar{I}}\right), \quad I \geq 0
$$

then averaging Eq. (23) over the distributions in Eq. (24) and using the Craig form of the Gaussian $Q$-function [6], we obtain after some routine integral evaluations the average BEP:

$$
P_{b}(E)=2 \int_{0}^{\pi / 2} \frac{2 \sin ^{2} \theta}{\bar{\gamma}} \exp \left(\frac{2 \sin ^{2} \theta}{\bar{\gamma}}\right) Q^{2}\left(\sqrt{\frac{2 \sin ^{2} \theta}{\bar{\gamma}}}\right) d \theta
$$

where $\bar{\gamma}=\bar{I}^{2} E_{b} / N_{0}$ is the total bit-energy-to-noise spectral density ratio in the presence of the turbulence. Using the asymptotic (large argument) form of the Gaussian $Q$-function, it is straightforward to show that $P_{b}(E)$ approaches a value of $1 / 2$ as $\bar{\gamma} \rightarrow 0$. Similarly, for large $\bar{\gamma}$, using the Gaussian $Q$-function approximation for small arguments, namely $Q(x) \cong(1 / 2) \exp \left(-x^{2} / 2\right)$, it is straightforward to show that the average BEP behaves asymptotically as $1 / 2 \bar{\gamma}$. At first glance, this asymptotic behavior might seem surprising in that it appears to imply that the modified Alamouti code does not provide a diversity of order 2. However, in the absence of Alamouti coding (i.e., no space diversity) and in the presence of the assumed exponential distribution modeling of the fading due to turbulence, the average BEP varies as $1 / \sqrt{\bar{\gamma}}$. Thus, there is in fact a diversity of order 2 when using the modified Alamouti code together with OOK or binary PPM.

We conclude by noting that the evaluation of the performances for other statistical irradiance models, more than one receive antenna, and combinations of trellis coding with the modified Alamouti block code are straightforward based on the vast literature available for the RF application.

\section{References}

[1] S. M. Alamouti, "A Simple Transmitter Diversity Scheme for Wireless Communications," IEEE J. Select. Areas Commun., vol. 1, pp. 1451-1458, October 1998.

\footnotetext{
${ }^{5}$ Note that we have intentionally avoided the use of the term "SNR" here since in the optical literature the quantity $\sqrt{E_{b} / N_{0}}$ is often defined as SNR [3] whereas in the RF literature SNR ordinarily refers to $E_{b} / N_{0}$.
} 
[2] L. Andrews, R. L. Phillips, and C. Y. Hopen, Laser Beam Scintillation with Applications, SPIE Press, 2001.

[3] R. M. Gagliardi and S. Karp, Optical Communications, New York: Wiley, 1976.

[4] A. Abdi and M. Kaveh, "K Distribution: An Appropriate Substitute for RayleighLognormal Distribution in Fading-Shadowing Wireless Channels," Electron. Letters, vol. 34, pp. 851-852, April 30, 1998.

[5] M. K. Simon, "A Moment Generating Function (MGF)-Based Approach for Performance Evaluation of Space-Time Coded Communication," Wireless Comm. and Mobile Computing, vol. 2, New York: John Wiley \& Sons, Ltd., pp. 667-692, 2002.

[6] J. W. Craig, "A New, Simple and Exact Result for Calculating the Probability of Error for Two-Dimensional Signal Constellations," IEEE MILCOM'91 Conference Rec., Boston, Massachusetts, pp. 25.5.1-25.5.5, November 4-7, 1991. 